\title{
A Prioritized Research Agenda for Using the Harmonized Outcome Measures To Support Patient- Centered Outcomes Research in Depression
}




\section{A Prioritized Research Agenda for Using the Harmonized Outcome Measures To Support Patient- Centered Outcomes Research in Depression}

\section{Prepared for:}

Agency for Healthcare Research and Quality

U.S. Department of Health and Human Services

5600 Fishers Lane

Rockville, MD 20857

www.ahrq.gov

Contract No. 75Q80119C00005

Prepared by:

OM1, Inc.

Investigators:

Michelle B. Leavy, M.P.H.

Anna Swenson, M.P.H.

Richard Gliklich, M.D.

AHRQ Publication No. 21(22)-EHC029

October 2021 
This report is based on research conducted by OM1, Inc. under contract to the Agency for Healthcare Research and Quality (AHRQ), Rockville, MD (Contract No. 75Q80119C00005). This work is supported by the Office of the Secretary Patient-Centered Outcomes Research Trust Fund under Interagency Agreement 18-596R-18. The findings and conclusions in this document are those of the authors, who are responsible for its contents; the findings and conclusions do not necessarily represent the views of AHRQ. Therefore, no statement in this report should be construed as an official position of AHRQ or of the United States (U.S.) Department of Health and Human Services.

None of the investigators have any affiliations or financial involvement that conflicts with the material presented in this report.

The information in this report is intended to help healthcare decision makers - patients and clinicians, health system leaders, and policymakers, among others-make well-informed decisions and thereby improve the quality of healthcare services. This report is not intended to be a substitute for the application of clinical judgment. Anyone who makes decisions concerning the provision of clinical care should consider this report in the same way as any medical reference and in conjunction with all other pertinent information, i.e., in the context of available resources and circumstances presented by individual patients.

This report is made available to the public under the terms of a licensing agreement between the author and AHRQ. This report may be used and reprinted without permission except those copyrighted materials that are clearly noted in the report. Further reproduction of those copyrighted materials is prohibited without the express permission of copyright holders.

AHRQ appreciates appropriate acknowledgment and citation of its work. Suggested language for acknowledgment: This work was based on a Research White Paper, A Prioritized Research Agenda for Using the Harmonized Outcome Measures To Support Patient-Centered Outcomes Research in Depression, by OM1, Inc., through the Agency for Healthcare Research and Quality (AHRQ).

Suggested citation: Leavy MB, Swenson A, Gliklich R. A Prioritized Research Agenda for Using the Harmonized Outcome Measures To Support Patient-Centered Outcomes Research in Depression. (Prepared OM1, Inc. under Contract No. 75Q80119C00005.) AHRQ Publication No. 21(22)-EHC029. Rockville, MD: Agency for Healthcare Research and Quality; October 2021. DOI: 10.23970/AHRQEPCWHITEPAPERDEPRESSIONRESEARCH. Posted final reports are located on the Effective Health Care Program search page. 


\section{Contents}

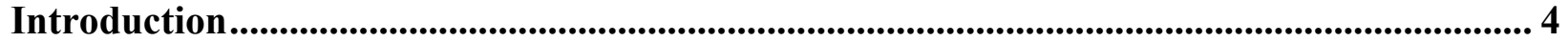

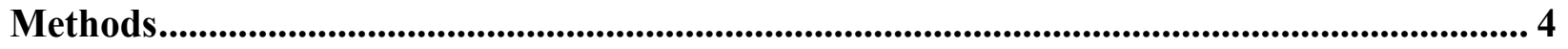

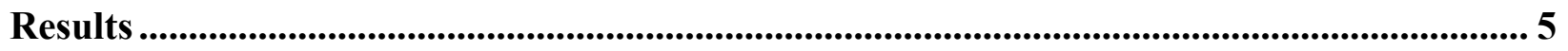

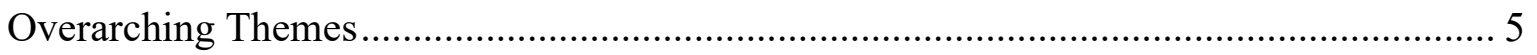

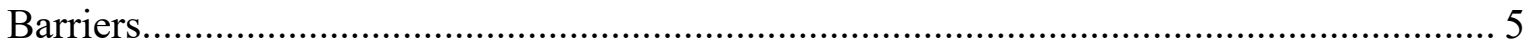

Roadmap for Depression PCOR Using Harmonized Outcome Measures.......................... 6

References........................................................................................................................ 16

Figures

Figure 1. Roadmap for implementing the research agenda .............................................. 7

\section{Tables}

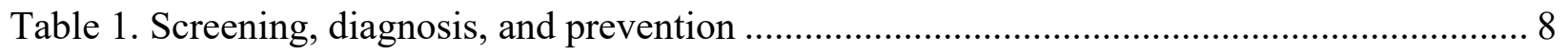

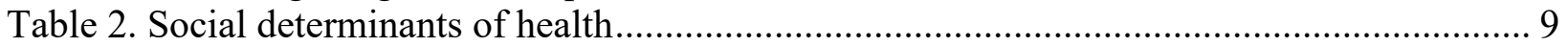

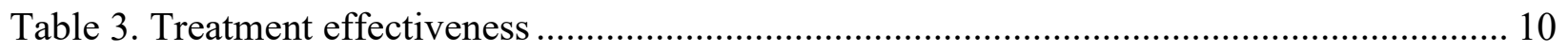

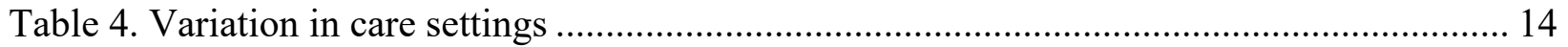

\section{Appendixes}

Appendix A. Research Questions Identified Through Literature Review and Stakeholder Discussions 


\section{Introduction}

A key objective of the Outcome Measure Harmonization and Data Infrastructure for PatientCentered Outcomes Research in Depression project is to demonstrate that the harmonized outcome measures are useful for research purposes. Many questions exist about depression treatment and outcomes. Linkage of data across studies and organizations using the harmonized outcome measures may provide a useful new resource to address at least some of these questions.

The goal of this project was to develop and prioritize a list of research topics related to depression care that can be addressed using the recently published harmonized depression outcome measures.

The specific objectives of the project were:

1. Develop a list of patient-centered outcomes research (PCOR) questions related to depression care that can be addressed using the harmonized depression outcome measures

2. Prioritize the list of research questions to provide a roadmap for using the harmonized depression outcome measures to address unmet research needs

3. Identify barriers to using the harmonized outcome measures to address the prioritized research topics

This purpose of this document is to present a research agenda and roadmap for addressing critical PCOR questions related to depression diagnosis, treatment, and outcomes that could be addressed using the harmonized outcome measures.

\section{Methods}

The project team conducted a horizon scan of the literature focusing on systematic reviews, research agendas, treatment guidelines, and prioritization research published from 2015 to the present. In addition, the team conducted searches of relevant websites (Agency for Healthcare Research and Quality, American Psychiatric Association, American Board of Family Medicine, American College of Physicians, Patient-Centered Outcomes Research Institute, and the U.S. Preventive Services Task Force) to identify documents that may not have been indexed on PubMed.

The following themes of interest were identified by reviewing the research gaps or future research sections of the documents identified in the search:

- Treatment Effectiveness

- Variation across Care Settings

- Screening, Diagnosis, and Prevention

- Treatment Resistant Depression

- Impact of Race, Ethnicity, Culture and Other Factors on Outcomes

- Depression and Comorbidities

- Perinatal and Postpartum Depression

- Suicidality

Specific patient-centered topics and questions of interest that could be addressed using the harmonized outcome measures in depression were then developed with reference to the previously reviewed research priorities and evidence gaps. Participants in the Stakeholder Panel were also asked to contribute priority research topics during the quarterly meeting, and those suggestions were incorporated into the list of potential questions (Appendix A). The Stakeholder 
Panel discussed the questions and provided feedback through a series of virtual meetings in 2020.

\section{Results}

Key points that emerged from the Panel discussion were as follows:

- Many of the questions relate to screening and diagnosis or effectiveness of treatment. For example, the questions in the Depression and Comorbidities theme largely relate to the effectiveness of treatment in different subpopulations. Reorganization of the questions into four overarching themes would reduce redundancy and improve the clarity of the research agenda.

- The role of measurement-based care should be incorporated into this research framework. When examining treatment effectiveness, it is critical to understand the framework in which care was provided - in other words, were the principles of measurement-based care followed?

- It is possible to answer some of these questions immediately using existing data infrastructure and methods. Other questions, however, require the development of new research methods or the expansion of data infrastructure. Prioritization therefore must reflect both the importance of the question and the interim steps necessary to address the question.

Based on the Stakeholder Panel's feedback, the questions were reorganized into four overarching themes, and many questions were revised to reflect the feasibility of addressing the question as well as subquestions of interest. Questions were also revised to be more specific and more appropriate for translation into a research study. The resulting research agenda presents a roadmap with short-term and long-term goals for addressing important PCOR questions related to depression treatment and outcomes.

\section{Overarching Themes}

PCOR questions related to depression screening, diagnosis, treatment, and outcomes that can be studied using the harmonized depression outcomes measures are grouped into four overarching themes:

1. Screening, Diagnosis, and Prevention

2. Social Determinants of Health

3. Treatment Effectiveness

4. Variation Across Care Settings

Questions related to treatment-resistant depression, the impact of race, ethnicity, culture, and other factors on outcomes, depression and comorbidities, perinatal and postpartum depression, and suicide thoughts, behaviors, and suicide risk were organized into these four themes.

\section{Barriers}

Specific barriers to addressing the questions were identified. These barriers broadly fell into two categories: data infrastructure gaps and lack of methods frameworks. For some questions, the necessary data are not captured systematically currently, making it challenging to address the question using observational data sources. For example, information on social determinants of 
health is often not documented or is included only in unstructured fields in electronic health records (EHRs), making it difficult to extract these data and examine their impact on depression screening, diagnosis, treatment, and outcomes. Further work is needed to expand the existing data infrastructure - meaning data captured through EHRs, patient registries, and other data collection systems - to capture these data in consistent, usable formats so they can be extracted for research purposes and used in the clinical workflow.

For other questions, new methods are needed. For example, there is interest in using patientreported tools to capture side effects related to treatment, but existing tools have not been adopted in clinical practice. As a result, there are questions about how often to capture this information and how to use the information to inform clinical decision-making. Further work is needed to create a framework for the systematic collection and use of patient-reported side effect information in the clinical workflow and for research purposes.

\section{Roadmap for Depression PCOR Using Harmonized Outcome Measures}

While some PCOR questions can be addressed using the current data infrastructure and harmonized outcome measures, other important PCOR questions must be addressed after data infrastructure has been created and/or new methods have been developed. To reflect this, the research agenda organizes questions into short-term PCOR questions, long-term PCOR questions, data infrastructure questions, and methods development questions. A roadmap was also created to show the process of addressing the PCOR priorities. The roadmap reflects an iterative process of addressing barriers to answering specific questions, refining questions, and ultimately addressing the full range of complex questions that need to be answered in order to improve depression outcomes (Figure 1).

The PCOR questions that make up the research agenda are organized according to theme and presented below (Tables 1-4). Each question is categorized as to whether it can be addressed now with existing data and the harmonized outcomes measures (short-term), whether it is an intermediary methods or data infrastructure question that needs to be addressed to remove a barrier to addressing a research question, or whether it is a PCOR question that cannot yet be answered with available data and measures (long-term). Some PCOR questions are both shortand long-term questions, in that the question can be answered now but may need to be revisited once additional data and/or methods are available. 


\section{Figure 1. Roadmap for implementing the research agenda}

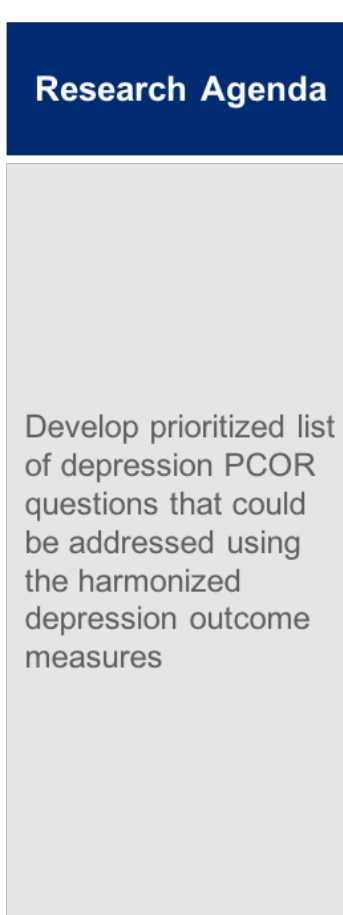

\begin{tabular}{|l|}
\hline \multicolumn{1}{|c|}{$1-3$ Years } \\
\hline $\begin{array}{l}\text { Conduct research } \\
\text { studies to answer } \\
\text { questions that can be } \\
\text { addressed } \\
\text { immediately }\end{array}$ \\
\hline $\begin{array}{l}\text { Develop methods to } \\
\text { measure additional } \\
\text { constructs }\end{array}$ \\
\hline $\begin{array}{l}\text { Expand data } \\
\text { infrastructure to } \\
\text { capture additional } \\
\text { relevant data (e.g., } \\
\text { social determinants of } \\
\text { health) }\end{array}$ \\
\hline
\end{tabular}

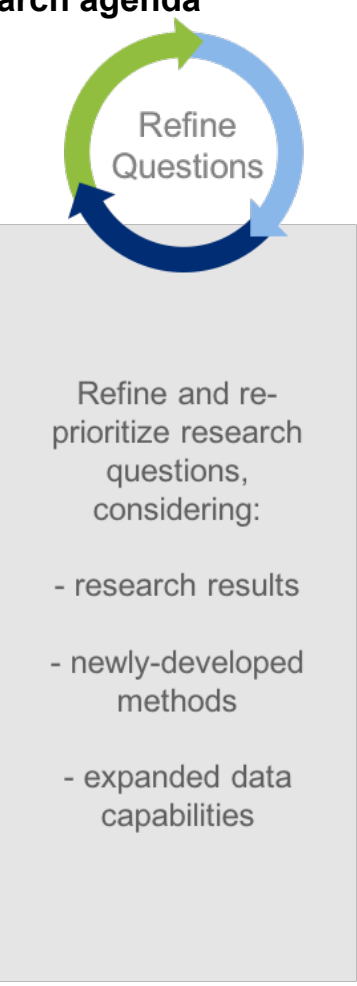

\begin{tabular}{|l|}
\hline \multicolumn{1}{|c|}{$3-5$ Years } \\
\hline $\begin{array}{l}\text { Answer questions } \\
\text { with newly available } \\
\text { data/methods }\end{array}$ \\
\hline $\begin{array}{l}\text { Validate methods } \\
\text { and/or develop } \\
\text { additional methods to } \\
\text { measure new } \\
\text { concepts }\end{array}$ \\
\hline $\begin{array}{l}\text { Expand data } \\
\text { infrastructure in line } \\
\text { with expanded } \\
\text { methods }\end{array}$ \\
\hline
\end{tabular}

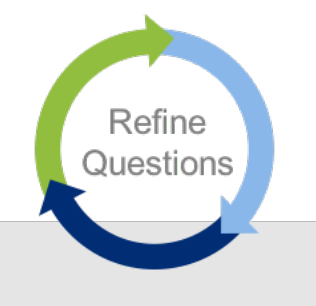

Continue to update, refine, and prioritize questions based on:

- depression research landscape

- study results

- improved data availability
5-10 Years

Answer complex questions about

treatment patterns effectiveness and

patient characteristics, and outcomes utilizing expanded data

\section{PCOR = patient-centered outcomes research}


Table 1. Screening, diagnosis, and prevention

Broad Question

Does screening for depression in primary care affect patient outcomes, such as remission and response?
Specific Questions

1. Compared with patients who were not screened for depression in a primary care setting, do those who were screened:

- Receive care in a different setting (e.g., primary care versus mental health)?

Differ in terms of type of treatment received?

Have significantly different time to initiation of treatment after diagnosis?
Question Type

Patient-centered

outcomes research

(short-term)

2. Do patients with no prior depression diagnosis or treatment who are screened for depression in a primary care setting differ from those who were not screened for depression with regards to depression outcomes, including:

Response rates (at 6 and 12 months)

Remission rates (at 6 and 12 months)

Rate of suicide ideation and behavior at 12 months

Patient-centered outcomes research (short-term)

3. Do patients with no prior depression diagnosis or treatment who are screened for depression in a primary care setting differ from those who were not screened for depression with regards to mortality rate (all-cause and death from suicide)?

Data infrastructure

1. Do particular subgroups (pregnant/postpartum women, those with comorbidities, certain age groups, patients belonging to particular race/ethnicity/socioeconomic groups) who are screened for depression in a primary care setting differ from those who were not screened for depression with regards to depression outcomes, including:

Response rates at 6 and 12 months

Remission rates at 12 months

Rate of suicide ideation and behavior at twelve months

2. Do particular subgroups (pregnant/postpartum women, those with comorbidities, certain age groups, patients belonging to particular race/ethnicity/socioeconomic groups) with no prior depression diagnosis or treatment who are screened for depression in a primary care setting differ from those who were not screened for depression with regards to mortality rate (all-cause and death from suicide)?

What are the optimal screening intervals across populations and in subpopulations?
1. Do response rate at 6 months and remission rate at 12 months vary between patients who are screened annually compared with those who are screened more frequently?
Patient-centered

outcomes research

(short-term)

\section{Data infrastructure} atient-centered outcomes research (short-term) 
2. Within subpopulations at higher risk of depression, such as postpartum women, patients with comorbidities such as cancer, etc., do those screened more frequently (e.g., every 3 months, every 6 months versus every 12 months) differ from those were not according to response rate and remission rates?

Patient-centered outcomes research (short-term)

Table 2. Social determinants of health

Broad Question

How can social determinants of health (SDOH) be measured in a systematic way so that this information is available for studying depression outcomes?

What is the impact of gender, race, ethnicity, culture, level of education, socioeconomic status and other SDOH on treatment choices and effectiveness of treatment?
Specific Questions

\section{Question Type}

1. Can existing methods for measuring $\mathrm{SDOH}$ be used to capture and record these data in electronic health records and other systems?

Data infrastructure

2. If methods do not exist, can methods be developed that enable the systematic and valid Methods collection and recording of SDOH data?

development

1. Across patients with different $\mathrm{SDOH}$, are there variations in:

- Choice of treatment type (pharmacotherapy, psychotherapy, complementary and alternative medicine, vagus nerve stimulation, transcranial magnetic stimulation, electroconvulsive therapy)

Response, remission rate at 6 and 12 months

Recurrence rate at 12 months

Rate of suicide ideation

2. Are there specific SDOH that are associated with higher rates of adverse events (AEs) from pharmacological treatments?

Patient-centered outcomes research (short \& long-term)

3. Are there specific AEs related to pharmacological treatment that occur more frequently in subgroups of interest?
Patient-centered outcomes research (short \& long-term) 
Table 3. Treatment effectiveness

Broad Question

What is the comparative effectiveness of treatments, augmentation strategies, and combination treatment

approaches?

\section{Specific Questions}

1. At 6 and 12 months after treatment initiation for each treatment/treatment strategy, what are the rates of:

- Response

Remission

Recurrence

2. At 6 and 12 months after treatment initation, how do the rates of response and remission differ across treatments/treatment strategies?

3. What is comparative effectiveness of pharmacological and nonpharmacological treatment interventions for adult patients with depression judged to be at imminent risk for suicide, in terms of reducing suicide ideation and behavior and death from suicide?

\section{Question Type}

Patient-centered outcomes research (short \& long-term)

Patient-centered outcomes research (short \& long-term)

Patient-centered outcomes research (short \& long-term)

4. How can we improve documentation and extraction of data on suicide thoughts, suicide behaviors, suicide risk, and death from suicide to increase opportunities for research using routinely recorded clinical data?

Data infrastructure

5. Are there variations in the rates of response, remission, recurrence, and adverse events across treatments/treatment strategies among subpopulations, such as:

\section{Older adults}

Patients with comorbid mental health conditions (e.g., anxiety, bipolar disorder)

Patients with other comorbid illnesses (e.g., cancer, cardiovascular disease, stroke,

other chronic medical conditions

Pregnant and postpartum women

6. Are there variations in patient-reported outcomes (PROs), such as depression-related quality of life and work productivity, across treatments/treatment strategies?

Patient-centered outcomes research (short \& long-term)

7. How are PROs (e.g., depression-related quality of life) captured and recorded in the electronic health record (EHR) so these data can be extracted for research purposes and made available to clinicians for decision making?

8. How can PROs (e.g., depression-related quality of life) be used within the clinical workflow to inform decision-making (thereby increasing collection and recording of these data so they can used for research purposes as well)?
Patient-centered

outcomes research

(short \& long-term)
Data infrastructure

Methods

development 
How should treatment be sequenced, specifically with differences across patient populations, and when should treatments be tapered or discontinued?

What are the long-term effects of treatment, including persistence of biological and psychological treatment effects?

1. Are there different rates of response/remission/recurrence between patients who start with pharmacotherapy versus psychotherapy versus a device versus CAM? Do these differences vary according to comorbidities and patient characteristics?

Patient-centered outcomes research (short-term)

2. Are there different rates of response/remission/recurrence between patients who begin with one class of anti-depressants versus another? Between different classes of second line treatment? Do these differences vary when adjusting for patient characteristics such as sex, race, age, or comorbidities?

\section{Patient-centered} outcomes research (short-term)

3. Is there an optimal length of time a patient should be in remission before treatment is tapered or discontinued?

What is the recurrence rate among patients who were in remission for 6 versus 12 months prior to discontinuation?

Patient-centered outcomes research (short-term)

4. Does the optimal timing of tapering/discontinuation vary according to patient characteristics or comorbidities?

- Are recurrence rates higher after tapering/discontinuation among certain patient populations? If so, does this vary according to the length of time a patient was in remission prior to discontinuation?

Patient-centered outcomes research (short-term)

1. Which specific data need to be collected to measure biological effects of treatment?

Data infrastructure

Patient-centered

outcomes research

(short \& long-term)

Patient-centered

outcomes research

(short \& long-term)

4. Which factors modify time in remission?

Age

Chronic medical conditions

Race/ethnicity

Patient-centered

outcomes research

$\mathrm{SDOH}$

(short \& long-term)

Pregnancy/postpartum status

1. What is the frequency and severity of side effects related to pharmacological/medical products and nonpharmacological treatment:

as reported in structured data in EHRs?
Patient-centered outcomes research (short-term) 
products and nonpharmacological treatment?
Which treatments/treatment approaches are most effective for patients who have not responded adequately to previous treatments? as extracted from unstructured fields in the EHR?

2. Do the frequency and severity of side effects vary by subpopulations, such as:

Older adults

Patients with comorbid mental health conditions (e.g., anxiety, bipolar disorder)

Patients with other comorbid illnesses (e.g., cancer, myocardial infarction, stroke

other chronic medical conditions)

Pregnant and postpartum women

3. Can side effect information be captured directly from patients and documented in the EHR in a systematic manner?

- How can the Frequency, Intensity, and Burden of Side Effects Rating (FIBSER) Scale

be used in a meaningful way in the clinical workflow? How often should it be collected? Can clinically significant cut-points be developed?

Methods

If FIBSER is found to not be feasible, can another validated questionnaire for collecting patient-reported AEs be developed and/or implemented?

4. What methods can be utilized to measure side effects of nonpharmacological depression treatments?

Methods

development

5. What is the relationship between suicidality and the use of antidepressant medications?

Patient-centered outcomes research (short \& long-term)

6. What is the relationship between antidepressant treatment and longer-term adverse outcomes, such as diabetes, decreased bone mineral density, and cardiovascular events?

Patient-centered outcomes research (short \& long-term)

1. In what ways is the construct of treatment-resistant depression (TRD) useful for identifying patients who have not responded adequately to previous treatment?
$\begin{array}{ll}\text { Is the TRD designation helpful in capturing the experience of people whose } & \text { Methods } \\ \text { symptoms continue to worsen, despite treatment? } & \text { development }\end{array}$
Methods
symptoms continue to worsen, despite treatment?
evelopment Are new constructs needed?

2. Can standardized definitions be developed and used for these constructs, so these patients can be identified consistently across data sources?

Data infrastructure

3. For patients who do not respond adequately to treatment, what is the impact of nonpharmacological treatment on health-related outcomes (quality of life and level of Patient-centered functional impairment), and how does this compare with pharmacological treatment? 


\begin{tabular}{|c|c|c|}
\hline Broad Question & Specific Questions & Question Type \\
\hline & $\begin{array}{l}\text { 4. Which psychological approaches and sequence of psychological treatments are most } \\
\text { effective for patients who do not respond adequately to treatment? }\end{array}$ & $\begin{array}{l}\text { Patient-centered } \\
\text { outcomes research } \\
\text { (short \& long-term) }\end{array}$ \\
\hline \multirow{3}{*}{$\begin{array}{l}\text { For relatively mild forms of } \\
\text { depression, how can patients } \\
\text { and their primary care providers } \\
\text { determine who should receive } \\
\text { "watchful waiting" (that is truly } \\
\text { watchful, consistent with } \\
\text { measurement-based care) and } \\
\text { who should receive active } \\
\text { intervention? }\end{array}$} & 1. How can data on watchful waiting be captured systematically in an EHR system? & Data infrastructure \\
\hline & $\begin{array}{l}\text { 2. Among patients with a Patient Health Questionnaire- } 9 \text { (PHQ-9) score of } 5-10 \text {, what is the } \\
\text { rate of response and of remission at } 6 \text { months and } 12 \text { months? }\end{array}$ & $\begin{array}{l}\text { Patient-centered } \\
\text { outcomes research } \\
\text { (long-term) }\end{array}$ \\
\hline & $\begin{array}{l}\text { 3. Do the response rate and remission rate differ between patients with a PHQ-9 score of 5- } \\
10 \text { who received watchful waiting versus active intervention? }\end{array}$ & $\begin{array}{l}\text { Patient-centered } \\
\text { outcomes research } \\
\text { (long-term) }\end{array}$ \\
\hline
\end{tabular}


Table 4. Variation in care settings

Are there differences between patients receiving care for depression in primary care versus mental health care settings?

\section{Are there any differences in} treatment effectiveness according to care setting or care model (e.g., collaborative care, co-location of mental health services in the primary care setting)?
1. Are there differences between patients receiving care for depression in primary care versus mental health care settings with regards to:

- Demographics

Frequency of PHQ-9 administration

Specific agents prescribed for pharmacotherapy, and sequencing of agents

Severity of symptoms, as measured by total PHQ-9 score $\mathrm{SDOH}$

1. Are there differences in the proportion of patients experiencing remission or response to their first line treatment, in the proportion of patients experiencing recurrence, and in proportion of patients reporting suicide ideation between the different care settings, after adjusting for potential confounders?

2. Do rates of remission, response, recurrence, and adverse events differ according to the care model in place at the primary care site, even after accounting for depression severity and other potential confounding factors?

Patient-centered outcomes research (short-term)

Patient-centered outcomes research (short-term)

Patient-centered outcomes research (short-term)

3. Which healthcare delivery systems/settings of care contribute to producing positive outcomes in patients at imminent risk of suicide?

Patient-centered outcomes research (short \& long-term)

4. What methods can be used to assess the adequacy of implementation of care models? Methods

development

5. What methods can be used to assess barriers to implementation of measurement-based Methods or other effective care models?

development

6. Which specific data elements need to be captured in an EHR, registry, or other data collection system to describe the type of care model in place at a site and the quality of implementation of the care model?
Are there differences between patients receiving care for depression via telehealth and those receiving in-person care?
1. Are there differences between patients receiving care for depression via telehealth with regards to:

Demographics

Frequency of PHQ-9 administration

Specific agents prescribed for pharmacotherapy, and sequencing of agents

Severity of symptoms, as measured by total PHQ-9 score

Care setting (primary care versus mental health)
Patient-centered

outcomes research

(short \& long-term) 
2. Holding all other variations equal (e.g., care setting, patient characteristics, type of care provided), are there variations in rate of response and rate of remission between patients

Patient-centered who access telehealth for depression and patients who receive care in-person?

Pesearch

What data need to be collected so that the impact of telehealth on treatment outcomes

\section{can be studied?}

4. Are there methods to measure and describe quality of care provided via telehealth?

\section{Data infrastructure}

Methods

development 


\section{References}

1. American Psychiatric Association: Practice Guideline for the Treatment of Patients with Major Depressive Disorder. 2010.

https://psychiatryonline.org/pb/assets/raw/sit ewide/practice_guidelines/guidelines/mdd.p df. Accessed January 29, 2020.

2. Cleare A, Pariante CM, Young AH, et al. Evidence-based guidelines for treating depressive disorders with antidepressants: A revision of the 2008 British Association for Psychopharmacology guidelines. J Psychopharmacol. 2015 May;29(5):459-525. doi: 10.1177/0269881115581093. Epub 2015 May 12. PMID: 25969470.

3. Cuijpers P, Quero S, Dowrick C, Arroll B. Psychological Treatment of Depression in Primary Care: Recent Developments. Current Psychiatry Reports. 2019;21(12):129.

4. Davies P, Ijaz S, Williams CJ, Kessler D, Lewis G, Wiles N. Pharmacological interventions for treatment-resistant depression in adults. Cochrane Database Syst Rev. 2019;12(12):CD010557CD010557.

5. Dunlop BW. Evidence-Based Applications of Combination Psychotherapy and Pharmacotherapy for Depression. Focus (Am Psychiatr Publ). 2016;14(2):156-173.

6. Ferenchick EK, Ramanuj P, Pincus HA. Depression in primary care: part $1-$ screening and diagnosis. BMJ (Clinical research ed). 2019;365:1794.

7. Gartlehner G, Gaynes BN, Amick HR, et al. Comparative Benefits and Harms of Antidepressant, Psychological, Complementary, and Exercise Treatments for Major Depression: An Evidence Report for a Clinical Practice Guideline from the American College of Physicians. Ann Intern Med. 2016 Mar 1;164(5):331-41. doi: 10.7326/M15-1813. Epub 2015 Dec 8.
8. Gaynes BN, Lux L, Lloyd S, et al. Nonpharmacologic Interventions for Treatment-Resistant Depression in Adults. Comparative Effectiveness Review No. 33. (Prepared by RTI International-University of North Carolina (RTI-UNC) Evidence-based Practice Center under Contract No. 290-020016I.) AHRQ Publication No. 11-EHC056EF. Rockville, MD: Agency for Healthcare Research and Quality. September 2011. www.effectivehealthcare.ahrq.gov/rep orts/final.cfm.

9. Gong J, Simon GE, Liu S. Machine learning discovery of longitudinal patterns of depression and suicidal ideation. PloS one. 2019;14(9):e0222665-e0222665.

10. Haigh EAP, Bogucki OE, Sigmon ST, et al. Depression among older adults: a 20-year update on five common myths and misconceptions. Am J Geriatr Psychiatry. 2018;26(1):107-22.

11. Ijaz S, Davies P, Williams CJ, et al. Psychological therapies for treatmentresistant depression in adults. Cochrane Database Syst Rev. 2018;5(5):CD010558.

12. Machmutow K, Meister R, Jansen A, et al. Comparative effectiveness of continuation and maintenance treatments for persistent depressive disorder in adults. Cochrane Database Syst Rev. 2019;5(5):CD012855.

13. Major Depressive Disorder. Questions Submitted for Consideration by Workshop Participants. Washington DC: PatientCentered Outcomes Research Institute; 2015. https://www.pcori.org/sites/default/files/PC ORI-Submitted-Questions-MajorDepressive-Disorder-060915.pdf. Accessed March 12, 2021.

14. O'Connor E, Rossom RC, Henninger M, et al. Primary Care Screening for and Treatment of Depression in Pregnant and Postpartum Women: Evidence Report and Systematic Review for the US Preventive Services Task Force. JAMA. 2016;315(4):388-406. 
15. Ostuzzi G, Matcham F, Dauchy S, Barbui C, Hotopf M. Antidepressants for the treatment of depression in people with cancer.

Cochrane Database Syst Rev. 2018;4(4):CD011006-CD011006.

16. Pettman D, O’Mahen H, Skoog Svanberg A, et al. Effectiveness and acceptability of cognitive-behavioural therapy-based interventions for maternal peripartum depression: a systematic review, metaanalysis and thematic synthesis protocol. BMJ open. 2019;9(12):e032659-e032659.

17. Pollok J, van Agteren JE, Carson-Chahhoud KV. Pharmacological interventions for the treatment of depression in chronic obstructive pulmonary disease. Cochrane Database Syst Rev. 2018;12(12):CD012346.

18. Qaseem A, Barry MJ, Kansagara D. Clinical Guidelines Committee of the American College of P. Nonpharmacologic Versus Pharmacologic Treatment of Adult Patients With Major Depressive Disorder: A Clinical Practice Guideline From the American College of Physicians. Annals of internal medicine. 2016;164(5):350-359.

19. Ramanuj P, Ferenchick EK, Pincus HA. Depression in primary care: part 2management. BMJ. 2019;365:1835.

20. Sebastianski M, Gates M, Gates A, et al. Evidence available for patient-identified priorities in depression research: results of 11 rapid responses. BMJ Open. 2019;9(6):e026847.

21. Siu AL, Domingo KB, Grossman DC, et al. Screening for Depression in Adults: US Preventive Services Task Force Recommendation Statement. JAMA. 2016;315(4):380-7.

22. Watson LC, Amick HR, Gaynes BN, et al. Practice-based interventions addressing concomitant depression and chronic medical conditions in the primary care setting: a systematic review and meta-analysis. J Prim Care Community Health. 2013;4(4):294306. 


\section{Appendix A. Research Questions Identified Through Literature Review and Stakeholder Discussions}

\section{Screening, Diagnosis, and Prevention}

1. Does screening for depression in primary care affect patient outcomes, such as remission and response?

2. Does the effect of screening on patient outcomes vary across subpopulations?

3. What are the optimal screening intervals across populations and in subpopulations?

\section{Treatment Effectiveness}

1. What is the comparative effectiveness of treatments, augmentation strategies, and combination treatment approaches?

2. How should treatment be sequenced, specifically with differences across patient populations, and when treatments should be tapered or discontinued?

3. What factors can be used to match patients with optimal therapies, particularly as it relates to selection of a first-line therapy, sequencing of treatment, and use of combination therapy?

4. What is the frequency and severity of side effects related to pharmacological and nonpharmacological treatment?

5. What is the relationship between tardive dysphoria and the resurgence of depression?

6. What is the relationship between antidepressant treatment and longer-term outcomes, such as diabetes, decreased bone mineral density, and cardiovascular events?

7. What are the long-term effects of treatment, including persistence of biological and psychological treatment effects?

8. For relatively mild forms of depression, how can patients and their primary care providers determine who should receive "watchful waiting" (that is truly watchful, consistent with measurement-based care) and who should receive active intervention?

\section{Variation Across Care Settings}

1. Are there differences between patients receiving care for depression in primary care versus mental health care settings with regards to demographics, severity of symptoms, types of treatments and outcomes?

2. Are there differences in the specific agents prescribed for pharmacotherapy, and in the sequencing of these agents?

3. Are there differences in the proportion of patients experiencing remission or response to their first line treatment, in the proportion of patients experiencing recurrence, and in time from diagnosis to response between the different care settings?

4. What is the comparative effectiveness of collaborative care versus integrated care or colocation of mental health services on depression outcomes in patients with depression in the primary care setting?

5. What proportion of primary care physicians follow guidelines for referring patients for mental health services, and how do outcomes differ for patients who meet the criteria for referral but continue to be managed in primary care versus those who go on to receive specialized care? 


\section{Treatment-Resistant Depression (TRD)}

1. What are the comparative tolerability and side effect profiles for nonpharmacological and pharmacological interventions in adults with TRD?

2. Which psychological approaches are most effective for TRD?

3. Which sequence of psychological treatments is most effective for TRD?

4. Is there a benefit to switching to psychotherapy versus remaining on antidepressant therapy for TRD?

5. Does the effectiveness of nonpharmacologic treatments vary based on symptom subtypes, sociodemographic characteristics, or co-existing medical conditions (perinatal, poststroke, post-myocardial infarction)?

6. What is the impact of nonpharmacological treatment of TRD on health-related outcomes (quality of life and level of functional impairment), and how does this compare with pharmacological treatment?

7. What is the relationship of social and demographic factors (socioeconomic status, gender, etc.) to the occurrence of TRD and treatment outcomes in TRD?

8. What is the comparative effectiveness of treatment approaches for severe TRD (defined as lack of response after at least 3 evidence-based interventions)?

9. What are the longer term outcomes of treatments for TRD?

\section{Impact of Race, Ethnicity, Culture and Other Factors on Outcomes}

1. What is the impact of gender, race, ethnicity, culture, level of education and socioeconomic status on treatment choices and response, and does the effectiveness of treatment vary based on these subgroups?

2. What is the impact of gender, race, ethnicity, culture, level of education and socioeconomic status on the adverse effects of pharmacological treatments, across subgroups and especially in older adults with comorbidities?

\section{Depression and Comorbidities}

1. What is the effectiveness of antidepressants in treating cancer patients with comorbid depression?

2. What is the comparative effectiveness of treatment approaches for adults who experience depression following a significant medical event such as myocardial infarction or stroke?

3. What is the comparative effectiveness of treatment approaches for adults with chronic medical conditions and comorbid depression?

4. What is the comparative effectiveness of pharmacotherapies, psychotherapies, and combination treatments for comorbid anxiety and depression?

5. What is the most effective treatment approach for patients who have mixed affective features (bipolar spectrum)? Do these patients benefit from mood stabilizers either instead of or in addition to antidepressants?

\section{Perinatal and Postpartum Depression}

1. What is the comparative effectiveness of psychotherapy versus pharmacotherapy or the combination of medication and psychotherapy during pregnancy and the post-partum period?

2. What are the clinical moderators of effectiveness of psychological interventions for postpartum depression?

3. What are the risks versus benefits of antidepressant therapy in pregnant and postpartum women? 


\section{Suicidality}

1. What are the comparative benefits and harms (risks) of pharmacological and nonpharmacological treatment interventions for adult patients with major depressive disorder judged to be at imminent risk for suicide?

2. Which healthcare delivery systems/settings of care contribute to producing positive outcomes in patients at imminent risk of suicide?

3. What is the relationship between suicidality and the use of antidepressant medications?

4. What are effective approaches to stratifying the risk of suicide in patients who are depressed and present with suicidal ideation in the primary care setting? 